\title{
Entanglement of Open Quantum Systems in Noninertial Frames
}

\author{
Salman Khan ${ }^{\dagger *}$ M. K. Khan ${ }^{\ddagger}$ \\ $\dagger$ Department of Physics, COMSATS Institute of Information \\ Technology, Islamabad, Pakistan \\ $\ddagger$ Department of Physics, Quaid-i-Azam University, \\ Islamabad, Pakistan
}

August 18, 2018

\begin{abstract}
We study the effects of decoherence on the entanglement generated by Unruh effect in accelerated frames by using various combinations of an amplitude damping channel, a phase damping channel and a depolarizing channel in the form of multilocal and collective environments. Using concurrence as entanglement quantifier, we show that the occurrence of entanglement sudden death (ESD) depends on different combinations of the channels. The ESD can be avoided under a particular configuration of the channels. We show that the channels can be used to distinguish between a moving and a stationary frame.
\end{abstract}

PACS: 03.65.Ud; 03.65.Yz; 03.67.Mn;04.70.Dy

Keywords: Entanglement; Decoherence; Noninertial frames.

\section{Introduction}

The last two decades has witnessed the rapid development in the field of quantum information and strengthened the notion that entanglement is not only one of the fundamental concepts of quantum theory but also a key concept for transmitting and processing quantum information [1. Entanglement between spatially separated parties is used as a potential source for quantum teleportation of unknown states [2, quantum key distribution [3], quantum cryptography [4] and quantum computation [5, 6. However, the behavior of entanglement between various systems is still not fully known and efforts are on the line to understand its dynamics deeper under various setups. The study of entanglement in a bipartite system has recently been extended to the relativistic setup and its behavior for various fields has been examined [7, 8, 9, 10, 11, 12. However,

\footnotetext{
*sksafi@phys.qau.edu.pk
} 
these investigations on entanglement are limited to closed quantum systems. Practically, quantum systems are influenced by its environment that may results in non-unitary dynamics of the system. For most accurate and practical results in quantum processing, the effect of environment on the entanglement between spatially separated systems needs to be necessarily investigated. The environmental effect on a quantum system gives rise to the phenomenon of decoherence that causes an irreversible transfer of information from the system to the environment [13, 14, 15].

Alsing et al [7] have shown that the entanglement between two modes of a free Dirac field is degraded by the Unruh effect and asymptotically reaches a nonvanishing minimum value in the infinite acceleration. In Ref. [16, the dynamics of entanglement for Dirac field in the presence of Unruh effect has been studied under amplitude damping channel. The effect of decoherence on the entanglement of Dirac field using a phase damping channel, a depolarizing channel and a phase flip channel has been investigated in Ref. [17.

In this paper we investigate the effect of decoherence on the entanglement of Dirac field in a noninertial system by considering various combinations of an amplitude damping, a phase damping and a depolarizing channel in the form of multilocal, collective and global noises. For example, we consider that if one qubit is locally coupled to an amplitude damping channel and the other is locally coupled to a phase damping channel then both the qubits are collectively coupled to a depolarizing channel. Using concurrence as the entanglement quantifier, we show that the rate of decrease of concurrence depends on the coupling of the kind of a channel to a particular qubit. For example, under the influence of multilocal coupling when Alice's qubit is influenced by phase damping channel and Rob's qubit is influenced by amplitude damping channel, the entanglement sudden death (ESD) [18] occurs earlier for large acceleration of the frames under some particular conditions. On the other hand, Under some other setups, the ESD can be avoided or delayed or even faster.

We rigorously consider three different cases. In one case we allow Rob's qubit to interact locally with an amplitude damping channel and Alice's qubit interact locally with a phase damping channel. In the second case, the coupling of these two channels are reversed in the sense that Rob's qubit interacts with a phase damping channel and Alice's qubit interacts with an amplitude damping channel. However, in both these cases the two qubits are coupled collectively with a depolarizing channel. In the third case, the two channels in the multilocal coupling of the qubits are depolarizing and phase damping channels while collectively the two qubits are coupled to an amplitude damping channel.

We consider that Alice and Rob, the two parties, share the following maximally entangled state at a point in flat Minkowski spacetime

$$
|\psi\rangle_{M}=\frac{1}{\sqrt{2}}\left(\left|0_{\omega_{A}}\right\rangle_{M}\left|0_{\omega_{R}}\right\rangle_{M}+\left|1_{\omega_{A}}\right\rangle_{M}\left|1_{\omega_{R}}\right\rangle_{M}\right) .
$$

In Eq. (11) $|0\rangle$ and $|1\rangle$ kets represent, respectively, the vacuum and excited states. The first entry in each pair of the kets is in Alice's possession and the second entry is in Rob's possession. The subscirpts $\omega_{N}(N=A, R)$ of the kets reflect 
that we consider the initial entanglement between these two modes and all the rest modes are in vacuum states. Furthermore, we suppose that each player has a device that is capabale of decting only the mode that she/he has shared for the initial entanglement. Rob then moves with a constant acceleration and Alice stays stationary. For accelerated observer the suitable coordinates are Rindler coordinates. These coordinates define two different regions, usually denoted by $I$ and $I I$, and are called Rindler regions. An observer in one region is causally disconnected from the other region. In other words, an observer in region $I$ has no access to information that leaks out to region $I I$ and vice versa (for detail see [7] and reference therein). A given Minskowski mode of a particular frequency spreads over all positive Rindler frequencies that peaks about the Minskowski frequency [19, 20]. However, to simplify our problem we consider a single mode only in the Rindler region $I$, which is valid if the observers' detectors are highly monochromatic that detects the frequency $\omega_{A} \sim \omega_{B}=\omega$. From this point onward, with this approximation, the frequency subscript of kets will be dropped.

From the accelerated Rob's frame, the Minkowski vacuum state is found to be a two-mode squeezed state [7,

$$
|0\rangle_{M}=\cos r|0\rangle_{I}|0\rangle_{I I}+\sin r|1\rangle_{I}|1\rangle_{I I}
$$

where $\cos r=\left(e^{-2 \pi \omega c / a}+1\right)^{-1 / 2}$. The constant $\omega, c$ and $a$, in the exponential stand, respectively, for Dirac particle's frequency, the speed of light in vacuum and Rob's acceleration. In Eq. (2) the subscripts $I$ and $I I$ of the kets represent the Rindler modes in region $I$ and $I I$, respectively. The excited state in Minkowski spacetime is related to Rindler modes as follow [7]

$$
|1\rangle_{M}=|1\rangle_{I}|0\rangle_{I I}
$$

In terms of Minkowski modes for Alice and Rindler modes for Rob, the maximally entangled initial state of Eq. (11) by using Eqs. (2) and (3) becomes

$$
|\psi\rangle_{A, I, I I}=\frac{1}{\sqrt{2}}\left(\cos r|0\rangle_{A}|0\rangle_{I}|0\rangle_{I I}+\sin r|0\rangle_{A}|1\rangle_{I}|1\rangle_{I I}+|1\rangle_{A}|1\rangle_{I}|0\rangle_{I I}\right) \text {. }
$$

Traditionally, we consider Rob to be in region $I$, then, he is causally disconnected from region $I I$. All the modes in region $I I$ of Eq. (4) needs to be discard. So, tracing over all the modes in region $I I$ leaves the following mixed density matrix between Alice and Rob

$$
\begin{aligned}
\rho_{A, I}= & \frac{1}{2}\left[\cos ^{2} r|00\rangle_{A, I}\langle 00|+\cos r\left(|00\rangle_{A, I}\langle 11|+| 11\rangle_{A, I}\langle 00|\right)\right. \\
& \left.\sin ^{2} r|01\rangle_{A, I}\langle 01|+| 11\rangle_{A, I}\langle 11|\right] .
\end{aligned}
$$

\section{The system in noisy environment}

The effect of decoherence is studied via quantum channels in the Kraus operators formalism. The Kraus operators for the three channels of a single qubit system 
Table 1: A single qubit Kraus operators for amplitude damping channel, phase damping channel and depolarizing channel.

\begin{tabular}{|c|cc}
\hline amplitude damping & $E_{o}=\left(\begin{array}{cc}1 & 0 \\
0 & \sqrt{1-p_{1}}\end{array}\right)$, & $E_{1}=\left(\begin{array}{cc}0 & \sqrt{p_{1}} \\
0 & 0\end{array}\right)$ \\
\hline phase damping & $E_{o}=\left(\begin{array}{cc}1 & 0 \\
0 & \sqrt{1-p_{2}}\end{array}\right)$, & $E_{1}=\left(\begin{array}{cc}0 & 0 \\
0 & \sqrt{p_{2}}\end{array}\right)$ \\
\hline \multirow{2}{*}{ depolarizing } & $E_{o}=\sqrt{1-p_{3}}\left(\begin{array}{cc}1 & 0 \\
0 & 1\end{array}\right)$, & $E_{1}=\sqrt{p_{3} / 3}\left(\begin{array}{cc}0 & 1 \\
1 & 0\end{array}\right)$, \\
& $E_{2}=\sqrt{p_{3} / 3}\left(\begin{array}{cc}0 & -i \\
i & 0\end{array}\right)$, & $E_{3}=\sqrt{p_{3} / 3}\left(\begin{array}{cc}1 & 0 \\
0 & -1\end{array}\right)$ \\
\hline
\end{tabular}

that we use in this paper are given in Table 1 . When a single qubit system evolves under the action of amplitude damping channel there is a probability to change the state of the system from state $|1\rangle$ to state $|0\rangle$. When the density matrix of a single qubit system is influenced by a phase damping channel, the diagonal elements remains unaffected while the off-diagonal elements decay. On the other hand, the study of the effect of a depolarizing noise on a quantum system is important because a pure state of a system when influenced by such a noise is replaced, with some probability, with a maximally mixed state. In Table 1, the action of the Kraus operators of each channel is parameterized by the decoherence parameters $p_{i}(i=1,2,3)$ whose values lie between 0 and 1 . For $p_{i}=0$, the channels have no effect on the system and for $p_{i}=1$, the system is fully decohered. Furthermore, the Kraus operators for each channel obey the completeness relation $\sum_{i} E_{i}^{\dagger} E_{i}=I$. The evolution of the initial density matrix of the system when it is influenced by the global environment is given as follow

$$
\rho_{f}=\sum_{i} \sum_{j} \sum_{k}\left(E_{i}^{A R} E_{j}^{R} E_{k}^{A}\right) \rho_{A, I}\left(E_{k}^{A \dagger} E_{j}^{R \dagger} E_{i}^{A R \dagger}\right),
$$

where $E_{k}^{A}=E_{m}^{A} \otimes I_{2}, E_{j}^{R}=I_{2} \otimes E_{n}^{R}$ are the Kraus operators of the local coupling of Alice's qubit and Rob's qubit, respectively. The $I_{2}$ is identity operator and the subscripts $m$ and $n$ represent the number of single qubit Kraus operators of the particular channel that is coupled to Alice's qubit or Rob's qubit. The $E_{i}^{A R}$ are the Kraus operators for the case of collective coupling and are formed from all the possible combinations of the tensor product of the Kraus operators of a single qubit channel in the form $E_{q}^{A} \otimes E_{q}^{R}$. The subscripts $q$, represents the number of Kraus operators for a one qubit channel used as a collective environment. The system is said to be coupled with multilocal environment when both the qubits are influenced by their local environments and it is said to be under the action of global environment when both multilocal and collective couplings are switched on simultaneously (global = multilocal + collective). First we consider the situation in which Alice qubit is locally influenced by phase damping channel, Rob's qubit is locally influenced by amplitude damping channel and the two qubits are then coupled collectively to depolarizing channel. Under such condition, the summations over $i, j$ and $k$ in Eq. (6) are, respectively, given by 
$i=0,1,2, \ldots 16$ and $j, k=0,1$. If $p_{1}, p_{2}$ and $p_{3}$ stand for decoherence parameters of amplitude damping channel, phase damping channel and depolarizing channel, respectively, then the non-zero elements of the final density matrix of the system in the light of Eq. (6) become

$$
\begin{aligned}
\rho_{11}= & \frac{1}{36}\left(-8 p_{3}^{2}\left(p_{1}-1\right)-\left(2 p_{3}\left(4 p_{3}-9\right)+9\right)\left(p_{1}-1\right) \cos 2 r\right. \\
& \left.-6 p_{3}\left(p_{1}+1\right)+9\left(p_{1}+1\right)\right), \\
\rho_{14}= & \frac{1}{18}\left(3-4 p_{3}\right)^{2} \sqrt{\left(-1+p_{1}\right)\left(-1+p_{2}\right)} \cos r \\
\rho_{22}= & \frac{1}{36}\left(9+8 p_{3}^{2}\left(-1+p_{1}\right)-9 p_{1}+6 p_{3}\left(1+p_{1}\right)\right. \\
& \left.+\left(9+2 p_{3}\left(-9+4 p_{3}\right)\right)\left(-1+p_{1}\right) \cos 2 r\right), \\
\rho_{33}= & \frac{1}{18}\left(p_{3}\left(9+4 p_{3}\left(-1+p_{1}\right)-15 p_{1}\right)+9 p_{1}\right. \\
& \left.+p_{3}\left(-3+4 p_{3}\right)\left(-1+p_{1}\right) \cos 2 r\right), \\
\rho_{41}= & \frac{1}{18}\left(3-4 p_{3}\right)^{2} \sqrt{\left(-1+p_{1}\right)\left(-1+p_{2}\right)} \cos 2 r \\
\rho_{44}= & \frac{1}{18}\left(9-9 p_{1}+p_{3}\left(-9-4 p_{3}\left(-1+p_{1}\right)+15 p_{1}\right)\right. \\
& \left.-p_{3}\left(-3+4 p_{3}\right)\left(-1+p_{1}\right) \cos 2 r\right) .
\end{aligned}
$$

The spin-flip matrix of the final density matrix of Eq. (6) is defined as $\tilde{\rho}_{f}=$ $\left(\sigma_{2} \otimes \sigma_{2}\right) \rho_{f}\left(\sigma_{2} \otimes \sigma_{2}\right)$, where $\sigma_{2}$ is the Pauli matrix. The degree of entanglement in the two qubits mixed state in a noisy environment can be quantified conveniently by concurrence $C$, which is given as 221,22$]$

$$
C=\max \left\{0, \sqrt{\lambda_{1}}-\sqrt{\lambda_{2}}-\sqrt{\lambda_{3}}-\sqrt{\lambda_{4}}\right\} \quad \lambda_{i} \geq \lambda_{i+1} \geq 0,
$$

where $\lambda_{i}$ are the eigenvalues of the matrix $\rho_{f} \tilde{\rho}_{f}$. The eigenvalues under the action of global environment become

$$
\begin{aligned}
\lambda_{1,2}= & \frac{1}{324}\left(54 p_{3}-36 p_{3}^{2}+81 p_{1}-216 p_{3} p_{1}+144 p_{3}^{2} p_{1}-81 p_{1}^{2}+216 p_{3} p_{1}^{2}\right. \\
& -144 p_{3}^{2} p_{1}^{2}+\left(3-4 p_{3}\right)^{2}\left(-1+p_{1}\right)\left(-24 p_{3}\left(-1+p_{2}\right)+16 p_{3}^{2}\left(-1+p_{2}\right)\right. \\
& \left.+9\left(-2+p_{1}+p_{2}\right)\right) \cos ^{2} r+2\left(3-4 p_{3}\right)^{2} p_{3}\left(-3+2 p_{3}\right)\left(-1+p_{1}\right)^{2} \cos ^{4} r \\
& \pm 2\left[( 3 - 4 p _ { 3 } ) ^ { 4 } ( - 1 + p _ { 1 } ) ( - 1 + p _ { 2 } ) \operatorname { c o s } ^ { 2 } r \left(-9\left(-6 p_{3}\left(1-2 p_{1}\right)^{2}\right.\right.\right. \\
& \left.+4 p^{2}\left(1-2 p_{1}\right)^{2}+9\left(-1+p_{1}\right) p_{1}\right)+9\left(3-4 p_{3}\right)^{2}\left(-1+p_{1}\right)^{2} \cos ^{2} r \\
& \left.\left.\left.+2\left(3-4 p_{3}\right)^{2} p_{3}\left(-3+2 p_{3}\right)\left(-1+p_{1}\right)^{2} \cos ^{4} r\right)\right)\right]^{1 / 2}, \\
\lambda_{3}= & \lambda_{4}=\frac{1}{1296}\left(3 \left(-96 p_{3}^{3}\left(-1+p_{1}\right)^{2}+32 p_{3}^{4}\left(-1+p_{1}\right)^{2}-54\left(-1+p_{1}\right) p_{1}\right.\right. \\
& \left.-6 p_{3}^{2}\left(-7+14 p_{1}+p_{1}^{2}\right)+9 p_{3}\left(5-10 p_{1}+13 p_{1}^{2}\right)\right)+2\left(3-4 p_{3}\right)^{2} \\
& \left(-1+p_{1}\right)\left(-6 p_{3}\left(-1+p_{1}\right)+4 p_{3}^{2}\left(-1+p_{1}\right)+9 p_{1}\right) \cos 2 r \\
& \left.+\left(3-4 p_{3}\right)^{2} p_{3}\left(-3+2 p_{3}\right)\left(-1+p_{1}\right)^{2} \cos 4 r\right),
\end{aligned}
$$


where in $\lambda_{1,2}$, the two eigenvalues are differentiated by \pm sign, respectively. Using Eq. (8) the concurrence can straightforwardly be calculated. It is important to note that the concurrence defined by the eigenvalues of Eq. (91) reduces to the result of Ref. [7] when the decoherence parameters $p_{i}=0$.

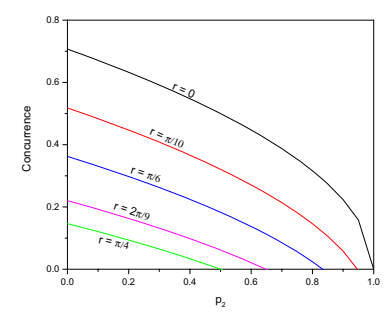

(a)

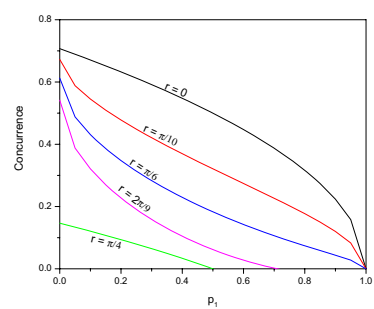

(b)

Figure 1: (color online) (a) The concurrence for different values of the acceleration is plotted against the decoherence parameter $p_{2}$, for the case when both the qubits are coupled to their respective environments in multilocal coupling. The other parameters are set to $p_{1}=0.5$, and $p_{3}=0$. (b) The concurrence against $p_{1}$ for $p_{2}=0.5$ and $p_{3}=0$ for different values of the acceleration of Rob's frame.

To see how the concurrence and hence the entanglement change when the system is coupled to noisy environments in the presence of Unruh effect, we plot the concurrence for each kind of the coupling of the three channels against the decoherence parameters for various values of the acceleration $r$ of the noninertial frame. In Fig. 1(a,b), the concurrence, when the collective environment is switched off $\left(p_{3}=0\right)$, is plotted for the case of multilocal coupling. Fig. $1 a$ shows the behavior of concurrence against the decoherence parameter $p_{2}$ (phase 
damping) for $p_{1}=0.5$ (amplitude damping). One can see that the concurrence is strongly acceleration dependent as the initial value of the concurrence decreases with increased acceleration. For a given acceleration, the decoherence parameter considerably damps the concurrence as the system evolves and it becomes zero at a definite value of the decoherence parameter (particular time) and hence loss of the entanglement occurs. The ESD occurs earlier for the case of larger acceleration. On the other hand, the behavior of concurrence is different when it is plotted against $p_{1}$ for a particular value of $p_{2}$. Fig. $1 b$ shows such a situation for $p_{2}=0.5$. Here the concurrence is not as much strongly acceleration dependent as in the case of Fig. 1a, except for the larger values of acceleration. For smaller acceleration of the noninertial frame, the ESD does not happen, the concurrence goes to zero only when the channel is fully decohered (infinite time). However, for large values of the acceleration $(r=\pi / 4)$, the concurrence is influenced identically by both $p_{1}$ and $p_{2}$. From the different behavior of concurrence and hence of ESD in the range of smaller acceleration, the two channels can be used to identify the stationary and the accelerated observers. The behavior of concurrence is shown in Fig. $2(a, b)$ for the case when both the collective and multilocal environments are simultaneously switched on, that is, when the system is coupled to global environment. In Fig. $2 a$, the values of decoherence parameters that parameterize the multilocal environment are set to $p_{1}=p_{2}=0.1$. It can be seen that the concurrence is strongly dependent both on acceleration and decoherence parameter. The ESD occurs faster than in the case when the system is coupled to multilocal environment only. Fig. $2 b$ shows the situation in which $p_{1}=p_{2}=p_{3}=p$. One can see from the figure that under such constraint, the concurrence, as compared to Fig. $2 a$, becomes more dependent on decoherence parameter and less dependent on the acceleration.

Now, we want to see the effect of interchanging multilocal channels that is, when Alice's qubit is locally coupled to an amplitude damping environment and Rob's qubit interacts locally with a phase damping environment. For this case, the summations over $i, j$ and $k$ in Eq. (6) remain unchanged. The concurrence can straightforwardly be calculated as in the previous case. Without writing its mathematical form, we prefer to show its behavior by plotting it against the decoherence parameters. The concurrence as a function of $p_{1}$ for $p_{2}=0.5$ is shown in Fig. 3 for different values of acceleration when the collective environment is switched off. The figure shows that the concurrence is significantly both acceleration and decoherence parameter dependent such that it decreases with the increasing values of both of these quantities. However, no ESD occurs for any value of the acceleration, the concurrence goes to zero for every acceleration only when $p_{1}=1$. Unlike the previous case, the plot of concurrence against $p_{2}$ is exactly the same as given in Fig. 3. This means that the rapid fall of concurrence in the previous case (Fig. 1a) is somehow compensated by the acceleration of the moving frame in this case. The two channels are not differentiable in this case. Nevertheless, ESD does occur in this case when the system evolves in the global environment. The effect of global environment on the concurrence is shown in Fig. 4. A comparison with Fig. $2 a$ shows that the concurrence is influenced almost identically in the two cases by the decoherence 
parameters, however, it is less affected by the acceleration in the later case.

Next, we consider the setup in which Alice's qubit interacts locally with a depolarizing channel and Rob's qubit interacts locally with a phase damping channel as before. The two qubits then interact collectively with an amplitude damping channel. The summations over $i, j$ and $k$ in Eq. (6) become $i=k=$ $0,1,2,3$ and $j=0,1$. Using Eq. (6) the final density matrix can easily be calculated. After finding the spin-flip matrix, the four eigenvalues of the matrix $\rho \tilde{\rho}$ are given by

$$
\begin{aligned}
\lambda_{1,2}= & \frac{1}{36}\left(-1+p_{1}\right)^{2}\left(18+9 p_{1}^{2}-9 p_{2}-36 p_{3}+12 p_{1} p_{3}\right. \\
& -12 p_{1}^{2} p_{3}+24 p_{2} p_{3}+20 p_{3}^{2}-8 p_{1} p_{3}^{2}+4 p_{1}^{2} p_{3}^{2}-16 p_{2} p_{3}^{2} \\
& \pm \sqrt{2}\left[-\left(-1+p_{2}\right)\left(3-4 p_{3}\right)^{2} \cos ^{2} r\left(-3+p_{3}+p_{3} \cos 2 r\right)\right. \\
& \times\left(-3\left(1+p_{1}+2 p_{1}^{2}\right)+2\left(-1+p_{1}\right)^{2} p_{3}+\left(-1+p_{1}\right)\right. \\
& \left.\left.\times\left(3+2\left(-1+p_{1}\right) p_{3}\right) \cos 2 r\right)\right]^{1 / 2}+\left(9\left(-2+p_{1}+p_{2}\right)\right. \\
& \left.+6\left(7-3 p_{1}+2 p_{1}^{2}-4 p_{2}\right) p_{3}-8\left(3-2 p_{1}+p_{1}^{2}-2 p_{2}\right) p_{3}^{2}\right) \sin ^{2} r \\
& \left.+2\left(-1+p_{1}\right) p_{3}\left(3+2\left(-1+p_{1}\right) p_{3}\right) \sin ^{4} r\right) \\
\lambda_{3}= & \lambda_{4}=\frac{1}{144}\left(-1+p_{1}\right)^{2}\left(3 \left(2 p_{3}^{2}\left(-1+p_{1}\right)^{2}+6 p_{1}\left(1+2 p_{1}\right)\right.\right. \\
& \left.+p\left(1+7 p_{1}-8 p_{1}^{2}\right)\right)+2\left(4 p_{3}^{2}\left(-1+p_{1}\right)^{2}-9 p_{1}\right. \\
& \left.\left.-12 p\left(-1+p_{1}\right) p_{1}\right) \cos 2 r+p_{3}\left(3+2 p_{3}\left(-1+p_{1}\right)\right)\left(-1+p_{1}\right) \cos 4 r(f) 10\right)
\end{aligned}
$$

The concurrence can be found by using these eigenvalues in Eq. (8). To study the influence of the noisy environment on the concurrence, we proceed by plotting it against the decoherence parameters as done in the previous cases. First we consider the effects of decoherence in the multilocal coupling. The plot of concurrence against $p_{3}\left(p_{2}=0.2\right)$ and $p_{2}\left(p_{3}=0.2\right)$ for $p_{1}=0$ is shown in Fig. $5(a, b)$. Though the behavior of concurrence is different in both of these figures, however, the ESD cannot be avoided. The ESD in this case occurs faster as compared to the previous two cases. On the other hand, the damping of concurrence due to acceleration is stronger in Fig. $5 b$ than in Fig. $5 a$. Finally we show the behavior of concurrence when the system is coupled with the global environment. Fig. 6 shows the concurrence of the system against $p_{1}$ for different values of acceleration with $p_{2}=p_{3}=0.2$. It is obvious from the last two figures that when one qubit is locally coupled to depolarizing channel there is no way to avoid ESD and it happens faster than the other two cases.

\section{Conclusion}

In conclusion, we have studied the behavior of entanglemnent of Dirac fields by considering different combinations of the three different quantum channels. We show that under multilocal environment when Alice's qubit is coupled to a phase damping channel and Rob's qubit is coupled to an amplitude damping channel, the ESD may or may not occur. However, the distinguishing effects 
of the two channels may be used to identify which of the observer is in motion. On the other hand, if the multilocal coupling of the two channels with two qubits is interchanged, the two channels affect the concurrence identically and cannot be distinguished from each other. However, the ESD can be avoided. The concurrence goes to zero only when one of the channels is fully decohered. Furthermore, it is shown that irrespective of the multilocal coupling's configuration, the ESD occurs when the collective environment is present. Moreover, It is shown that ESD occurs faster and can not be avoided when one of the two qubits is locally coupled to the depolarizing channel.

\section{References}

[1] Jeager G 2007 Quantum Information an overview (Springer Science+Business Media, New York).

[2] Bennett C H, Brassard G, Crepeau C, Jozsa R, Peres A, and Wootters W K 1993 Phys. Rev. Lett. 701895.

[3] Ekert A 1991 Phys. Rev. Lett. 67661

[4] Bennett C H, Brassard G and Mermin N D 1992 Phys. Rev. Lett. 68557

[5] Grover L K 1997 Phys. Rev. Lett. 79325

[6] DiVincenzo D P 1995 Science 270255

[7] Alsing P M, Fuentes-Schuller I, Mann R B and Tessier T E 2006 Phys. Rev. A 74032326

[8] Yi Ling et al 2007 J. Phys. A: Math. Theor. 409025

[9] Gingrich R M and Adami C 2002 Phys. Rev. Lett. 89270402

[10] Pan Q and Jing J 2008 Phys. Rev. A 77024302

[11] Fuentes-Schuller I and Mann R B 2005 Phys. Rev. Lett. 95120404

[12] Terashima H and Ueda M 2003 Int. J. Quantum Inf. 193

[13] Zurek W H et al 1991 Phys. Today 4436

[14] Breuer H P and Petruccione F 2002 The Theory of Open Quantum Systems (Oxford University Press, Oxford); Carmichael H 1993 An Open Systems Approach to Quantum Optics (Springer, Berlin,).

[15] Zurek W H 2003 Rev. Mod. Phys. 75715

[16] Wang J and Jing J arxiv: 1005.2865v4 (2010).

[17] S. Khan and M. K. Khan 2011 J. Phys. A: Math. Theor. 44045305 
[18] Yu T and Eberly J H 2004 Phys. Rev. Lett. 93 140404; 2006 Opt. Commun. 264 393; 2006 Phys. Rev. Lett. 97 140403; 2007 Quantum Inf. Comput. 7 459; 2009323598.

[19] Takagi S 1986 Prog. Theor. Phys. Suppl. 881

[20] Alsing P M, McMahon D and Milburn G J 2004 J. Opt. B: Quantum Semiclass. Opt. 6834

[21] Wootters W K 1998 Phys. Rev. Lett. 802245

[22] Coffman V, Kundu J and Wootters W K 2000 Phys. Rev. A 61052306 


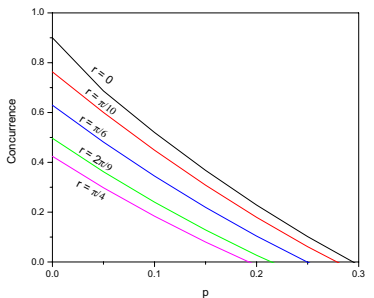

(a)

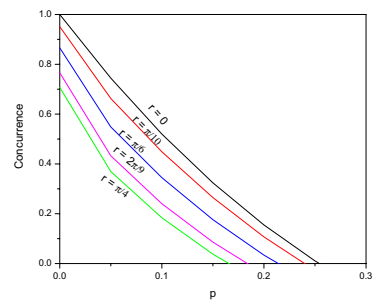

(b)

Figure 2: (color online) (a) The concurrence for different values of the acceleration is plotted against the decoherence parameter $p_{3}$, for the case when both the multilocal and collective environments are switched on. The other parameters are set to $p_{1}=p_{2}=0.1$. (b) The concurrence is plotted for different values of the acceleration against decoherence parameter $p$ under the conditions $p_{1}=p_{2}=p_{3}=p$. 


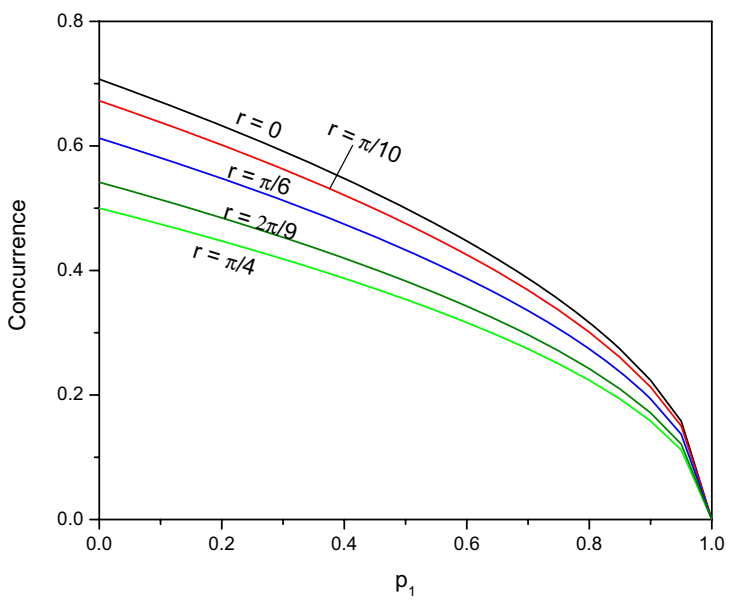

Figure 3: (color online) The concurrence for different values of the acceleration is plotted against the decoherence parameter $p_{1}$, for the case of multilocal coupling when Alice's qubit is coupled to amplitude damping channel and Rob's qubit to phase damping channel. The other parameters are set to $p_{2}=0.5$, and $p_{3}=0$. 


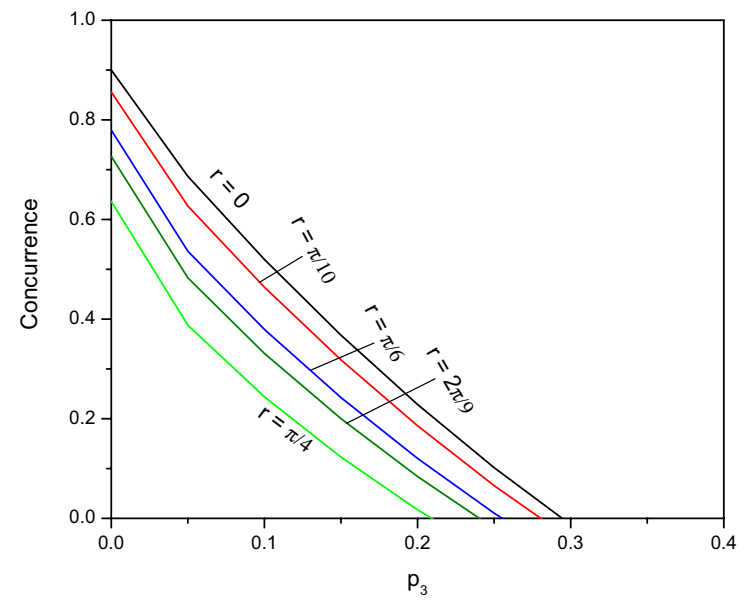

Figure 4: (color online) The concurrence for different values of the acceleration is plotted against the decoherence parameter $p_{3}$, for the case of global environment. The other parameters are set to $p_{1}=p_{2}=0.1$. 


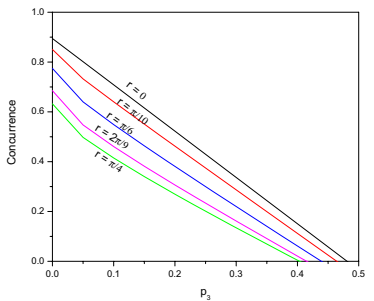

(a)

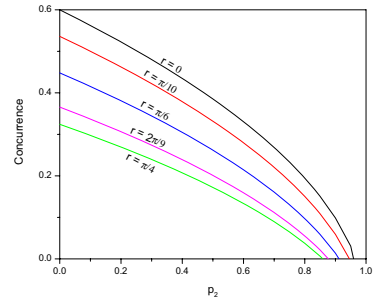

(b)

Figure 5: (color online) (a) The concurrence for different values of the acceleration is plotted against the decoherence parameter $p_{3}$, for the case when both the qubits are coupled to their respective environments in multilocal coupling. The other parameters are set to $p_{2}=0.2$, and $p_{1}=0$. (b) The concurrence against $p_{2}$ for $p_{3}=0.2$ and $p_{1}=0$ for different values of the acceleration of Rob's frame. 


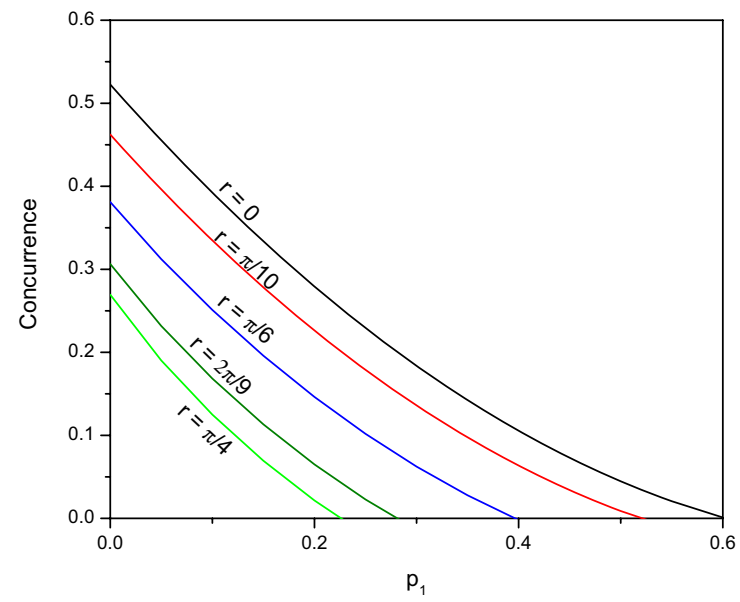

Figure 6: (color online) The concurrence for different values of the acceleration is plotted against the decoherence parameter $p_{1}$, for the case of global environment. The other parameters are set to $p_{2}=p_{3}=0.2$. 